\title{
Risk of Cancer in Cases of Suspected Lynch Syndrome Without Germline Mutation
}

\begin{abstract}
MARÍA RODRÍGUEZ-SOLER, ${ }^{1,2}$ LUCÍA PÉREZ-CARBONELL, ${ }^{2}$ CARLA GUARINOS, ${ }^{2}$ PEDRO ZAPATER, ${ }^{3}$ ADELA CASTILLEJO, ${ }^{4}$ VICTOR M. BARBERÁ, ${ }^{4}$ MIRIAM JUÁREZ, ${ }^{2}$ XAVIER BESSA, ${ }^{5}$ ROSA M. XICOLA, ${ }^{6}$ JUAN CLOFENT, ${ }^{7}$ LUIS BUJANDA, ${ }^{8}$ FRANCESC BALAGUER, ${ }^{9}$ JOSEP-MARIA REÑÉ, ${ }^{10}$ LUISA DE-CASTRO, ${ }^{11}$ JOSÉ C. MARÍN-GABRIEL, ${ }^{12}$ ANGEL LANAS, ${ }^{13}$ JOAQUÍN CUBIELLA, ${ }^{14}$ DAVID NICOLÁS-PÉREZ, ${ }^{15}$ ALEJANDRO BREA-FERNÁNDEZ, ${ }^{16}$ SERGI CASTELLVÍ-BEL, ${ }^{9}$ CRISTINA ALENDA, ${ }^{17}$ CLARA RUIZ-PONTE, ${ }^{16}$ ANGEL CARRACEDO, ${ }^{16}$ ANTONI CASTELLS, ${ }^{9}$ MONTSERRAT ANDREU, ${ }^{5}$ XAVIER LLOR, ${ }^{6}$ JOSÉ L. SOTO, ${ }^{4}$ ARTEMIO PAYÁ, ${ }^{17}$ and RODRIGO JOVER ${ }^{1}$

${ }^{1}$ Unidad de Gastroenterología, ${ }^{2}$ Unidad de Investigación, ${ }^{3}$ Clinical Pharmacology Department, and ${ }^{17}$ Pathology Department, Hospital General Universitario de Alicante, Alicante, Spain; ${ }^{4}$ Molecular Genetics Laboratory, Hospital General Universitario de Elche, Elche, Spain; ${ }^{5}$ Gastroenterology Department, Hospital del Mar, Barcelona, Spain; ${ }^{6}$ Department of Medicine and Cancer Center, University of Illinois at Chicago, Chicago, Illinois; ${ }^{7}$ Gastroenterology Department, Hospital de Sagunto, Sagunto, Spain; ${ }^{8}$ Gastroenterology Department, ClBERehd, Universidad del Pais Vasco, San Sebastian, Spain; ${ }^{9}$ Gastroenterology Department, Hospital Clínic, CIBERehd, IDIBAPS, University of Barcelona, Barcelona, Catalonia, Spain; ${ }^{10}$ Gastroenterology Department, Hospital Arnau de Vilanova, Lleida, Spain; ${ }^{11}$ Gastroenterology Department, Complexo Hospitalario de Vigo, Vigo, Spain; ${ }^{12}$ Gastroenterology Department, Hospital 12 de Octubre, Madrid, Spain;

${ }^{13}$ Gastroenterology Department, Hospital Clínico Lozano Blesa, Zaragoza, Spain; ${ }^{14}$ Gastroenterology Department, Complexo Hospitalario de Ourense, Ourense, Spain; ${ }^{15}$ Gastroenterology Department, Hospital Universitario de Canarias, Santa Cruz de Tenerife, Spain; and ${ }^{16}$ Fundación Pública Galega de Medicina Xenomica, Grupo de Medicina Xenómica, IDIS, CIBERER, Santiago de Compostela, Spain
\end{abstract}

This article has an accompanying continuing medical education activity on page e13. Learning Objective: Upon completion of this CME activity, successful learners will be able to assess the different diagnostic tests to establish a diagnosis of Lynch syndrome.

\section{See editorial on page 868 .}

BACKGROUND \& AIMS: Colorectal cancers (CRCs) with microsatellite instability (MSI) and a mismatch repair (MMR) immunohistochemical deficit without hypermethylation of the MLH1 promoter are likely to be caused by Lynch syndrome. Some patients with these cancers have not been found to have pathogenic germline mutations and are considered to have Lynch-like syndrome (LLS). The aim of this study was to determine the risk of cancer in families of patients with LLS. METHODS: We studied a population-based cohort of 1705 consecutive patients, performing MSI tests and immunohistochemical analyses of MMR proteins. Patients were diagnosed with Lynch syndrome when they were found to have pathogenic germline mutations. Patients with MSI and loss of MSH2 and/or MSH6 expression, isolated loss of PMS2 or loss of MLH1 without MLH1 promoter hypermethylation, and no pathogenic mutation were considered to have LLS. The clinical characteristics of patients and the age- and sex-adjusted standardized incidence ratios (SIRs) of cancer in families were compared between groups. RESULTS: The incidence of CRC was significantly lower in families of patients with LLS than in families with confirmed cases of Lynch syndrome (SIR for Lynch syndrome, 6.04; 95\% confidence interval [CI], 3.58-9.54; SIR for LLS, 2.12; 95\% CI, 1.16-3.56; $P<.001)$. However, the incidence of CRC was higher in families of patients with LLS than in families with sporadic CRC (SIR for sporadic CRC, $0.48 ; 95 \% \mathrm{CI}, 0.27-0.79 ; P<.001)$. CONCLUSIONS: The risk of cancer in families with LLS is lower that of families with Lynch syndrome but higher than that of families with sporadic CRC. These results confirm the need for special screening and surveillance strategies for these patients and their relatives.

Keywords: Inherited Colon Cancer; Cancer Risk; Genetic Testing; Immunohistochemistry.

I ynch syndrome (LS) is the most common inherited colon cancer susceptibility syndrome and is caused by germline mutations in one of several DNA mismatch repair (MMR) genes, mainly $M L H 1$ and $M S H 2$ but also MSH6 and PMS2. ${ }^{1-3}$ Patients with LS have an increased risk of colorectal cancer (CRC), endometrial cancer, and several other cancers, including ovarian, upper urinary tract, gastric, small bowel, biliary/pancreatic, skin, and brain cancers. The molecular signature of LS is microsatellite instability (MSI), which is found in more than 95\% of LS-associated CRCs. ${ }^{4}$ However, MSI is also present in up to $15 \%$ of sporadic CRCs due to hypermethylation of the promoter region of MLH1 in tumor cells. Immunohistochemical (IHC) studies of MMR proteins have been shown to be equivalent to MSI in detecting MMR-defective CRC. ${ }^{5}$ CRC with MSI and a lack of staining of MSH2, MSH6, or MLH1 without promoter hypermethylation is a strong indicator of $M S H 2, M S H 6$, or $M L H 1$ germline mu-

\footnotetext{
Abbreviations used in this paper: $\mathrm{Cl}$, confidence interval; CRC, colorectal cancer; IHC, immunohistochemical; LLS, Lynch-like syndrome; LS, Lynch syndrome; LSRC, Lynch syndrome-related cancer; MMR, mismatch repair; MSI, microsatellite instability; SIR, standardized incidence ratio.
}

\section{(1) 2013 by the AGA Institute $0016-5085 / \$ 36.00$}

http://dx.doi.org/10.1053/j.gastro.2013.01.044 
tations. ${ }^{6}$ However, some of these cases of CRC do not have pathogenic mutations in MMR genes. These cases are suspected to be nonsporadic because no mechanism of inactivation is known for these genes other than germline mutations in the context of LS. These patients are considered to have "probably nonsporadic" CRC or Lynchlike syndrome (LLS), and decisions about their management are not simple because of unconfirmed suspicions of hereditary cancer. These cases must be distinguished from familial CRC type $X$, in which tumors do not show MMR deficiency. No studies have characterized these patients with CRC, and the risk of cancer in this group of families is not known. Therefore, the surveillance strategy for these patients and their relatives is unclear.

We analyzed the clinical and familial characteristics of patients diagnosed with LLS, LS, or sporadic CRC. The main aim of this study was to determine the risk of cancer in families of patients with LLS.

\section{Patients and Methods Patients and Data Collection}

This population-based, observational, cohort study included 1705 patients with CRC from 2 nationwide multicenter studies: EPICOLON I and EPICOLON II. EPICOLON I included consecutive patients with a new diagnosis of CRC between November 2000 and October 2001 with the main goal of estimating the incidence of LS in Spain. ${ }^{7}$ EPICOLON II also included consecutive patients with newly diagnosed CRC between March 2006 and December 2007 with the aim of investigating different aspects related to the diagnosis of hereditary CRC. ${ }^{8}$ All of the patients provided written informed consent. Both studies were approved by the institutional review boards of the participating hospitals.

Patients were divided into 3 groups based on genetic data: (1) the LS group, in which patients had a confirmed pathogenic mutation in MLH1, MSH2, MSH6, or PMS2; (2) the LLS group, in which patients had MSI and loss of MSH2/MSH6 expression, isolated loss of PMS2, or loss of expression of MLH1 without MLH1 promoter hypermethylation in which no germline mutation was found; and (3) the sporadic group, in which patients with CRC and microsatellite stable tumors had normal expression of MMR genes or a loss of MLH1 expression with MLH1 promoter hypermethylation.

Demographic, clinical, and pathologic data were collected at the time of diagnosis. Cancer pedigrees were built at diagnosis for cases of CRC in the EPICOLON I and II studies. The pedigrees were traced backward and laterally as far as possible. This information was verified by reviewing medical records when available. Standardized incidence ratios (SIRs) for cancer were calculated as the ratio of the observed to expected number of cases diagnosed in the families at the time of inclusion in the EPICOLON I or II cohorts. To avoid recall bias, only cases of cancer in first-degree relatives were included in the calculation of SIR. We considered tumors in the endometrium, ovaries, upper urinary tract, stomach, small intestine, and hepatobiliary system as noncolorectal LS-related cancers (LSRCs). The index case was excluded for the analysis of family history at the time of diagnosis. Calculation of the SIR was only possible in families with complete pedigrees and information about the ages of all family members, including relatives without cancer.
In 2011, the pedigrees were updated by asking patients and/or relatives about new cases of cancer after diagnosis of the index case. We include the index case for this analysis, and the appearance of metachronous CRC or a new case of noncolorectal LSRC in the index case was considered a new case in the family.

\section{MSI, Immunohistochemical Staining, and Detection of Germline Mutations}

MSI analysis was performed in all patients. We ascertained MSI status using BAT26 and NR24 quasi-monomorphic markers as described previously. ${ }^{9}$ MSI was present when one of the 2 markers was unstable. IHC analysis of the $4 \mathrm{MMR}$ proteins MLH1, MSH2, MSH6, and PMS2 in tumor tissue was performed in all patients using tissue microarrays as described previously. ${ }^{10}$ In patients with a loss of MLH1, methylation of $M L H 1$ and BRAF mutation status was analyzed. MLH1 methylation analysis was performed using methylation-specific multiplex ligation-dependent probe amplification (MS-MLPA) according to the manufacturer's protocol using SALSA MS-MLPA Kit ME011 Mismatch Repair Genes (MRC-Holland, Amsterdam, The Netherlands). ${ }^{11}$ The V600E BRAF mutation was detected using specific TaqMan probes in real-time polymerase chain reaction (ABI Prism 7500; Applied Biosystems, Foster City, CA) and allelic discrimination software as described previously. ${ }^{12}$

Germline mutation analysis was performed in accordance with the results of IHC analysis as described previously. ${ }^{10} \mathrm{~Pa}$ tients with loss of MSH2 expression with no detected mutation were analyzed for EPCAM rearrangements using MLPA (MRCHolland) according to the manufacturer's recommended protocol. DNA sequencing was performed to characterize the deletion breakpoints. ${ }^{13}$ Large rearrangements (deletions and insertions) were tested using MLPA according to the manufacturer's protocol. The results of genetic analysis were interpreted based on the ACMG Recommendations for Standards for Interpretation of Sequence Variations (2000) and the InSIGHT database. ${ }^{14}$

\section{Statistical Analysis}

Continuous variables are reported as mean \pm standard deviation or median and 25th and 75th percentiles for nonnormally distributed data. Categorical variables are reported as frequencies or percentages. Significant differences between groups were analyzed using the $\chi^{2}$ test for categorical data and the non-parametric Mann-Whitney $U$ test for quantitative data.

The SIR of each cancer was calculated as the ratio of the observed to expected number of cases among relatives. Personyears were calculated from 20 years of age to the earliest cancer diagnosis or death. The expected number of cases was calculated as the sum of the products of the number of person-years for each 5-year age/sex group and the corresponding age/sex-specific incidence rates in Spanish regional registers. ${ }^{15}$ The confidence limits were based on Byar's approximation of the exact Poisson distribution, which is accurate even with small numbers. ${ }^{16}$ All reported $P$ values are 2 sided, and $P<.05$ was considered significant. All calculations were performed using SPSS 19.0 software (Chicago, IL).

\section{Results}

A total of 1705 patients with CRC were included in the study. The median age was 71 years (range, 27-101 years), and $59 \%$ of patients were male. Sixteen patients were excluded because of discrepancies between the IHC and MSI analyses; no mutation was found in these pa- 
Table 1. Comparison of Demographic, Clinical, and Pathologic Data from the LLS, LS, and Sporadic CRC Groups

\begin{tabular}{lccc}
\hline & LS $(\mathrm{n}=16)$ & LLS $(\mathrm{n}=43)$ & Sporadic CRC $(\mathrm{n}=1630)$ \\
\hline Female sex & $62.5(10)$ & $55.8(24)^{a}$ & $40.1(654)$ \\
Median age, $y$ (interquartile range) & $69(51-75)$ & $66(55-73)^{a}$ & $71(64-78)$ \\
Fulfilled revised Bethesda Guidelines & $81.3(13)$ & $51.2(22)^{a b}$ & $22.0(358)$ \\
Location, right colon & $56.3(9)$ & $55.8(24)^{a}$ & $26.9(438)$ \\
TNM stage II & $50.0(8)$ & $59.0(25)^{a}$ & $39.6(645)$ \\
Histology & & & \\
$\quad$ Poor differentiation & $25.0(4)$ & $4.9(2)^{b}$ & $8.3(135)$ \\
$\quad$ Lymphocytic infiltration & $25.0(4)$ & $28.6(12)$ & $29.2(475)$ \\
$\quad$ Mucinous & $20.0(3)$ & $36.6(15)^{a}$ & $13.0(212)$ \\
Metachronous CRC & $12.5(2)$ & $0(0)$ & $1.2(20)$ \\
Personal history of noncolorectal LS cancer & $43.8(7)$ & $11.6(5)^{a b}$ & $3.3(54)$ \\
Synchronous CRC & $12.5(2)$ & $9.3(4)$ & $5.6(91)$ \\
\hline
\end{tabular}

NOTE. All values are expressed as percent $(n)$ unless otherwise noted. ${ }^{a} P<.05$, patients with LLS versus patients with sporadic CRC.

${ }^{b} P<.05$, patients with LS versus patients with LLS.

tients. Therefore, data from 1689 patients were analyzed. Tumors from all patients were analyzed. A total of 135 patients $(8 \%)$ exhibited in their tumors MSI and loss of expression of any of the MMR proteins. In 104 patients (6.1\%), loss of MLH1 expression was found on IHC analysis. Of these patients, 25 (1.4\%) did not exhibit hypermethylation of the promoter region. We also used $B R A F$ mutation as a sporadic CRC marker in these 25 cases, but no case of $B R A F$ mutation indicating sporadic origin was found. Loss of MSH2 expression was seen in the IHC analysis of 22 patients (1.3\%). Three patients $(0.2 \%)$ had an isolated loss of MSH2 expression, and 19 (1.1\%) had a combined loss of MSH2 and MSH6. An isolated loss of MSH6 was found in 6 patients (0.3\%), and an isolated loss of PMS2 was found in 3 patients $(0.2 \%)$.

A germline pathogenic mutation in any of the MMR genes was found in 16 patients $(0.9 \%)$, who were considered to have LS. Three of these patients exhibited MSI with nonvaluable expression of MMR proteins on IHC analysis. Four of the patients with LS were found to have a pathogenic mutation in $M L H 1,8$ in $M S H 2,3$ in MSH6, and one in PMS2. All of these patients exhibited MSI. No case was found with deletions in EPCAM. Variants of uncertain significance were found in 5 patients (Supplementary Table 1). Forty-three patients $(2.5 \%)$ exhibited MSI and loss of MSH2/MSH6, PMS2, or MLH1 expression without promoter hypermethylation, but no pathogenic germline mutation was found. These patients were considered to have LLS. Among the patients with LLS, 21 were found to have loss of MLH1 protein expression and 22 loss of MSH2, MSH6, or PMS2 expression (14 with loss of MSH2 and MSH6, 6 with isolated loss of MSH6, and 2 with isolated loss of PMS2). Finally, 1630 patients (96\%) were considered to have sporadic CRC.

\section{Demographic, Clinical, and Pathologic Characteristics of Patients With LLS}

The characteristics of the LLS group $(n=43)$ were compared with those of the LS $(\mathrm{n}=16)$ and sporadic CRC $(\mathrm{n}=1630)$ groups $($ Table 1$)$. Fewer patients with
LLS fulfilled the revised Bethesda guidelines than patients with LS, and patients with LLS less often had a personal history of non-colorectal LSRC compared with patients with LS. There were no differences in the presence of metachronous CRC, median age at diagnosis of CRC, sex, and tumor characteristics, such as location or TNM classification. Patients with LLS were younger at diagnosis, predominantly female, and more frequently fulfilled the revised Bethesda guidelines compared with patients with sporadic CRC. A personal history of noncolorectal LSRC was more frequent in patients with LLS without differences in the presence of metachronous or synchronous CRC (Table 1).

\section{Risk of Familial Cancer}

A total of 13 families with LS and 25 families with LLS had complete pedigrees, including the ages of relatives without cancer. A random sample of 115 families with sporadic CRC was used for comparison. A total of 1102 first-degree relatives were included: 80 from families with LS, 177 from families with LLS, and 845 from families with sporadic CRC. The mean number of first-degree relatives was 6.1 for families with LS, 7.0 for families with LLS, and 7.3 for families with sporadic CRC.

In families with LS, we identified 18 cases of CRC and 6 cases of noncolorectal LSRC. There were no cases of cancer other than the index case in only 4 families (30.7\%). In families with LLS, we found 14 cases of CRC and 8 cases of noncolorectal LSRC. In 12 of 25 families (48\%), no case of cancer was found other than the index case. The characteristics of patients and distribution of cases of cancer in families with LLS are provided in Table 2. Finally, in families with sporadic CRC, 15 first-degree relatives had CRC and 27 had noncolorectal LSRC. No cases of cancer other than the index case were identified in 85 families $(79.9 \%)$.

The SIRs of CRC and noncolorectal LSRC are shown in Table 3. The incidence of CRC was significantly lower in families with LLS compared with confirmed families with LS (SIR in LS, 6.04; 95\% confidence interval [CI], 3.58 - 
Table 2. Characteristics and Family History of Patients With LLS

\begin{tabular}{|c|c|c|c|c|c|c|c|}
\hline Patient & Protein lost & $\begin{array}{c}\text { Age of index } \\
\text { case }(y)\end{array}$ & $\begin{array}{l}\text { No. of relatives } \\
\text { with CRC }{ }^{a} \\
(n=14)\end{array}$ & $\begin{array}{l}\text { No. of relatives with } \\
\text { noncolorectal LSRC } \\
\qquad(\mathrm{n}=8)\end{array}$ & $\begin{array}{l}\text { Total no. of first- } \\
\text { degree relatives } \\
\quad(n=177)\end{array}$ & $\begin{array}{c}\text { Cases of CRC } \\
(\%)^{c}\end{array}$ & $\begin{array}{c}\text { Cases of } \\
\text { noncolorectal } \\
\text { LSRC }(\%)^{d}\end{array}$ \\
\hline 1 & PMS2 & 71 & 0 & 1 (pancreas) & 10 & 0 & 10 \\
\hline 2 & MLH1/PMS2 & 81 & 0 & 0 & 9 & 0 & 0 \\
\hline 3 & MLH1/PMS2 & 73 & 0 & 0 & 7 & 0 & 0 \\
\hline 4 & MLH1/PMS2 & 75 & 1 & 1 (stomach) & 7 & 14.2 & 14.2 \\
\hline 5 & MLH1/PMS2 & 45 & 3 & 1 (ovary) & 10 & 30 & 10 \\
\hline 6 & $\mathrm{MSH} 2 / \mathrm{MSH} 6$ & 38 & 0 & 0 & 5 & 0 & 0 \\
\hline 7 & $\mathrm{MSH} 2 / \mathrm{MSH} 6$ & 74 & 0 & 0 & 6 & 0 & 0 \\
\hline 8 & $\mathrm{MSH} 2 / \mathrm{MSH} 6$ & 32 & 2 & 0 & 3 & 66.6 & 0 \\
\hline 9 & MLH1/PMS2 & 72 & 2 & 3 (uterus) & 11 & 18.1 & 27.2 \\
\hline 10 & MLH1/PMS2 & 51 & 0 & 0 & 4 & 0 & 0 \\
\hline 11 & MLH1/PMS2 & 46 & 1 & 1 (pancreas) & 3 & 33.3 & 33.3 \\
\hline 12 & $\mathrm{MSH} 2 / \mathrm{MSH} 6$ & 69 & 1 & 0 & 9 & 11.1 & 0 \\
\hline 13 & MSH6 & 55 & 0 & 0 & 6 & 0 & 0 \\
\hline 14 & MSH6 & 56 & 0 & 0 & 4 & 0 & 0 \\
\hline 15 & MLH1/PMS2 & 72 & 1 & 0 & 7 & 14.2 & 0 \\
\hline 16 & MSH2/MSH6 & 69 & 0 & 0 & 5 & 0 & 0 \\
\hline 17 & $\mathrm{MSH} 2 / \mathrm{MSH} 6$ & 66 & 0 & 1 (stomach) & 6 & 0 & 16.6 \\
\hline 18 & MSH2/MSH6 & 66 & 0 & 0 & 9 & 0 & 0 \\
\hline 19 & MLH1/PMS2 & 63 & 1 & 0 & 8 & 12.5 & 0 \\
\hline 20 & MLH1/PMS2 & 46 & 0 & 0 & 11 & 0 & 0 \\
\hline 21 & MLH1/PMS2 & 71 & 0 & 0 & 4 & 0 & 0 \\
\hline 22 & MLH1/PMS2 & 69 & 1 & 0 & 12 & 8.3 & 0 \\
\hline 23 & MLH1/PMS2 & 59 & 0 & 0 & 4 & 0 & 0 \\
\hline 24 & MSH6 & 79 & 1 & 0 & 8 & 12.5 & 0 \\
\hline 25 & MSH 6 & 54 & 0 & 0 & 9 & 0 & 0 \\
\hline
\end{tabular}

a Number of first-degree relatives diagnosed with CRC at any time until diagnosis of the index case.

${ }^{b}$ Number of first-degree relatives diagnosed with noncolorectal LSRC at any time until diagnosis of the index case.

'Percentage of family members diagnosed with CRC at any time until diagnosis of the index case.

'Percentage of family members diagnosed with noncolorectal LSRC at any time until diagnosis of the index case.

9.54; SIR in LLS, 2.12; 95\% CI, 1.16-3.56; $P<.001$ ). However, the incidence of CRC was significantly greater in the families with LLS than in the families with sporadic CRC (SIR in sporadic CRC, 0.48; 95\% CI, 0.27-0.79; $P<$ .001). The SIR for noncolorectal LSRC was not significantly higher in families with LS (SIR, 2.81; 95\% CI, 1.03-6.12) compared with families with LLS (SIR, 1.69; 95\% CI, 0.73-3.34; $P=.09$ ). There were no differences in the SIR for noncolorectal LSRC between families with LLS and families with sporadic CRC (SIR, 1.20; 95\% CI, $0.79-1.74 ; P=.5$ ). Taken together, the results indicate that, for CRC and noncolorectal LSRC, the highest risk is for families with LS (SIR, 4.69; 95\% CI, 3.00-6.98), followed by families with LLS (SIR, 1.94; 95\% CI, 1.22-2.94; $P<.001$ ). The risk in families with LLS was significantly higher than the risk in relatives of patients with sporadic CRC (SIR, 0.78; 95\% CI, 0.56-1.05; $P<.001$ ).
Figure 1 shows the cumulative age-of-onset curves for CRC among all relatives in the LS, LLS, and sporadic CRC groups. The relatives of patients with LLS developed CRC at an earlier mean age $(53.71 \pm 16.8$ years $)$ than those with sporadic CRC $(68.8 \pm 9$ years; $P=.004)$ but at a mean age similar to that in patients with LS (48.5 \pm $14.13 ; P=.23)$.

After a median of 8.3 years of prospective follow-up, cancer pedigrees were updated in 93 families: 10 in the LS group, 16 in the LLS group, and 67 in the sporadic CRC group. A total of 533 first-degree relatives were included (including the index case): 41 from families with LS, 89 from families with LLS, and 403 from families with sporadic CRC. During this period, 7 (17.1\%) new cases of CRC or non-colorectal LSRC appeared in families with LS, $4(4.5 \%)$ new cases in families with LLS, and 4 (0.9\%) new cases in families with sporadic CRC (Table 4).

Table 3. SIRs Between Families With LLS and Families With LS/Sporadic CRC

\begin{tabular}{|c|c|c|c|c|c|c|c|c|}
\hline & \multicolumn{2}{|c|}{ LS $(n=80)$} & \multirow{2}{*}{$\begin{array}{c}P \\
\text { value }^{a}\end{array}$} & \multicolumn{2}{|c|}{ LLS $(n=177)$} & \multirow{2}{*}{$\begin{array}{c}P \\
\text { value }^{b}\end{array}$} & \multicolumn{2}{|c|}{ Sporadic CRC $(n=845)$} \\
\hline & No. of tumors & SIR (95\% CI) & & No. of tumors & SIR (95\% CI) & & No. of tumors & SIR (95\% CI) \\
\hline CRC & 18 & $6.04(3.58-9.54)$ & $<.001$ & 14 & $2.12(1.16-3.56)$ & $<.001$ & 15 & $0.48(0.27-0.79)$ \\
\hline Non-CRC LSRC & 6 & $2.81(1.03-6.12)$ & .09 & 8 & $1.69(0.73-3.34)$ & .5 & 27 & $1.20(0.79-1.74)$ \\
\hline Total & 24 & $4.69(3.00-6.98)$ & $<.001$ & 22 & $1.94(1.22-2.94)$ & $<.001$ & 42 & $0.78(0.56-1.05)$ \\
\hline
\end{tabular}

${ }^{a}$ Comparing the SIR of the LS and LLS groups.

${ }^{b}$ Comparing the SIR of the LLS and sporadic CRC groups. 


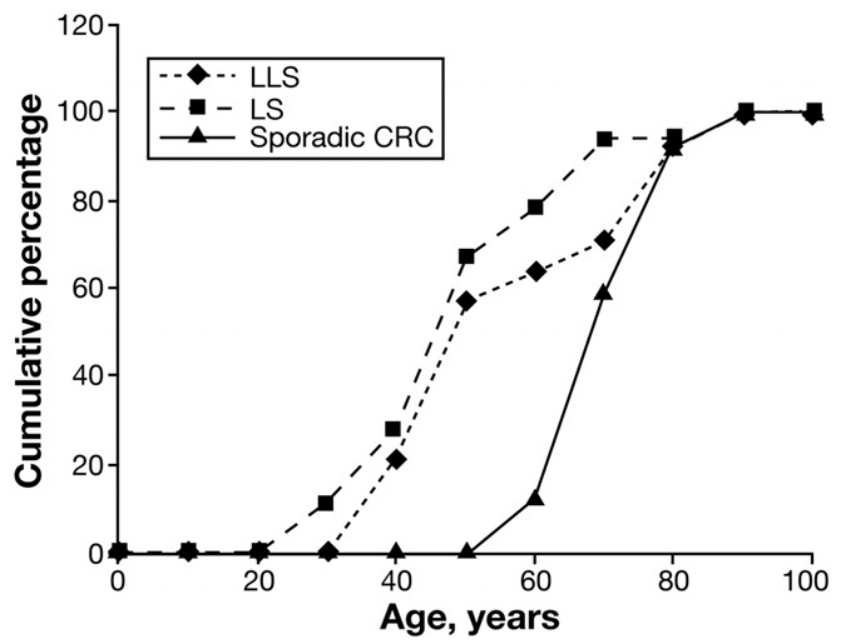

Figure 1. Cumulative age of onset of $C R C$ in first-degree relatives of patients with LS, LLS, and sporadic CRC.

\section{Discussion}

The main finding of our study was that the risk of CRC is lower in families with LLS than among patients with genetically confirmed LS but significantly higher than in cases of sporadic CRC. The results confirm the need for a special surveillance strategy for these patients and their relatives. In addition, the age of onset for CRC in families with LLS was similar to that for families with LS. Differences between families with LLS and families with sporadic CRC were more prominent in regard to risk of CRC than for risk of other noncolorectal LSRCs.

Recent studies have shown that MSI testing and IHC analysis of MMR genes in all patients with CRC improve the detection of patients with LS. ${ }^{10,17,18}$ Because of the generalization of this universal strategy following the Jerusalem guidelines, ${ }^{19}$ an increasing number of patients exhibit a loss of MMR protein expression with no pathogenic mutation. In cases of CRC with a loss of MSH2, MSH6, PMS2, or MLH1 without hypermethylation, no cause of MMR gene inactivation is known other than germline mutation. In these cases, when a germline mutation is not found, a high suspicion of LS persists, and these patients and their relatives should be followed up appropriately. The clinical characteristics of some of these patients suggest that they are clear hereditary cases, even though we were not able to find a genetic defect. Some of the pedigrees of patients with LLS showed a significant history of CRC with metachronous and synchronous tu- mors and fulfillment of the Amsterdam criteria. In these cases, the genetic defect was not found, probably because it was located in a still unknown part of the gene or simply because our technical capacity is not yet able to detect the pathogenic mutation. Some of these cases have been explained in the literature by alterations in other genes, such as in cases of EPCAM deletions or MLH1 constitutional epimutations. Other mechanisms, including inversions and duplications, could also explain some of these cases. ${ }^{20-26}$ However, some cases do not show any specific characteristics that suggest a hereditary origin. A notable proportion of families with LLS do not have a history of other cancers, and the only reason to suspect LS is the presence of MSI and loss of MMR protein expression. In these cases, determining the appropriate counseling for patients and their relatives is difficult. It is possible that some of these patients with LLS could be cases with CRC who may have false-positive results on IHC analysis and/or MSI or sporadic MMR-deficient CRC, and patients with LLS would be a mixture of patients with true LS with nondetected germline mutation and cases of sporadic CRC. However, the high risk of CRC found in our study suggests that, in its entirety, patients with LLS should be considered high-risk cases and strategies for prevention of cancer must be implemented in this group of patients and their relatives. The SIR of CRC for families with LLS was similar to that described in a group of families with familial colorectal cancer syndrome type $\mathrm{X}$, but in this syndrome no molecular alteration has been found. ${ }^{27}$ Even though LLS is a completely different entity because of the presence of MSI, the similar risk of CRC should guarantee at least a similar surveillance program, even in cases without a previous family history. Our results can contribute some rationale for designing follow-up strategies and, together with family history, can help clinicians appropriately schedule surveillance for these patients and their relatives. In our study, the age at onset of CRC was similar to that of LS, and therefore surveillance strategies should start at the same age as recommended for cases of LS. On the other hand, the frequency of CRC screening should be individualized. Given that the risk of CRC is lower in families with LLS than in families with LS, longer surveillance intervals for patients with LLS and relatives without a prominent family history of CRC may be recommended. We have not found a higher risk of noncolorectal LSRC in families with LLS compared with families with sporadic CRC. However, that can be attributable to the small num-

Table 4. Differences in the Prospective Appearance of New Cases of Cancer Between Families With LLS and Families With LS/Sporadic CRC During Follow-up

\begin{tabular}{lccccc}
\hline & LS $(\mathrm{n}=41)$ & $P$ value $^{a}$ & LLS $(\mathrm{n}=89)$ & $P$ value $^{b}$ & Sporadic CRC $(\mathrm{n}=403)$ \\
\hline CRC (\%) & $3(7.3)$ & .16 & $2(2.2)$ & .2 & $3(0.7)$ \\
Non-CRC LSRC (\%) & $4(9.8)$ & .05 & $2(2.2)$ & .02 & $1(0.2)$ \\
Total cancers (\%) & $7(17.1)$ & .01 & $4(4.5)$ & .01 & $4(0.9)$ \\
\hline
\end{tabular}

${ }^{a}$ Comparing the percentage in the LS and LLS groups.

${ }^{b}$ Comparing the percentage in the LLS and sporadic CRC groups. 
ber of cases detected in our series. For this reason, specific recommendations for endometrial and other noncolorectal LSRC cannot be appropriately supported by our data.

The limitations of our study are the possibility of underreporting or misreporting cancers because our information was not always confirmed with objective clinical and pathologic data. However, we believe this limitation is minor because it would affect the LLS group to the same extent as the other groups. Another limitation is the relatively small number of families, especially in the prospectively followed up cases, which precludes finding clear differences between groups. Moreover, the follow-up time for these cases could be considered too short.

The main strength of our study is its population-based approach, with cases ascertained from general clinics and not from specialized high-risk clinics. This approach provides robustness to our data in terms of potential applicability to general practice. The risk of cancer can be overestimated in studies from select populations in genetic high-risk clinics. Studies based on recruitment through cancer genetics clinics do not usually correct for the selection bias caused by the overrepresentation of families with multiple cases in the data set. ${ }^{28,29}$ Our results attempt to provide a rationale for follow-up and surveillance of this growing group of patients who will mostly be seen in general clinics and not in high-risk clinics. New research is necessary to refine the classification of these patients to distinguish between sporadic and true hereditary cases.

\section{Supplementary Material}

Note: To access the supplementary material accompanying this article, visit the online version of Gastroenterology at www.gastrojournal.org, and at http:// dx.doi:10.1053/j.gastro.2013.01.044.

\section{References}

1. Aarnio M, Sankila R, Pukkala E, et al. Cancer risk in mutation carriers of DNA-mismatch-repair genes. Int J Cancer 1999;81: 214-218.

2. Bonadona V, Bonaiti B, Olschwang S, et al. Cancer risks associated with germline mutations in MLH1, MSH2, and MSH6 genes in Lynch syndrome. JAMA 2011;305:2304-2310.

3. Watson P, Lynch HT. Extracolonic cancer in hereditary nonpolyposis colorectal cancer. Cancer 1993;71:677-685.

4. Aaltonen LA, Peltomaki P, Mecklin JP, et al. Replication errors in benign and malignant tumors from hereditary nonpolyposis colorectal cancer patients. Cancer Res 1994;54:1645-1648.

5. Pinol V, Castells A, Andreu M, et al. Accuracy of revised Bethesda guidelines, microsatellite instability, and immunohistochemistry for the identification of patients with hereditary nonpolyposis colorectal cancer. JAMA 2005;293:1986-1994.

6. Lynch HT, Boland CR, Rodriguez-Bigas MA, et al. Who should be sent for genetic testing in hereditary colorectal cancer syndromes? J Clin Oncol 2007;25:3534-3542.

7. Pinol V, Andreu M, Castells A, et al. Frequency of hereditary non-polyposis colorectal cancer and other colorectal cancer familial forms in Spain: a multicentre, prospective, nationwide study. Eur J Gastroenterol Hepatol 2004;16:39-45.

8. Abuli A, Bessa X, Gonzalez JR, et al. Susceptibility genetic variants associated with colorectal cancer risk correlate with cancer phenotype. Gastroenterology 2010;139:788-796.
9. Xicola RM, Llor X, Pons E, et al. Performance of different microsatellite marker panels for detection of mismatch repair-deficient colorectal tumors. J Natl Cancer Inst 2007;99:244-252.

10. Perez-Carbonell L, Ruiz-Ponte C, Guarinos C, et al. Comparison between universal molecular screening for Lynch syndrome and revised Bethesda guidelines in a large population-based cohort of patients with colorectal cancer. Gut 2012;61:865-872.

11. Perez-Carbonell L, Alenda C, Paya A, et al. Methylation analysis of MLH1 improves the selection of patients for genetic testing in Lynch syndrome. J Mol Diagn 2010;12:498-504.

12. Benlloch S, Paya A, Alenda C, et al. Detection of BRAF V600E mutation in colorectal cancer: comparison of automatic sequencing and real-time chemistry methodology. J Mol Diagn 2006; 8:540-543.

13. Van der KH, Wijnen J, Wagner A, et al. Molecular characterization of the spectrum of genomic deletions in the mismatch repair genes MSH2, MLH1, MSH6, and PMS2 responsible for hereditary nonpolyposis colorectal cancer (HNPCC). Genes Chromosomes Cancer 2005;44:123-138.

14. Ou J, Niessen RC, Vonk J, et al. A database to support the interpretation of human mismatch repair gene variants. Hum Mutat 2008;29:1337-1341.

15. Chirlaque-Lopez MD, Salmeron-Martinez D, Valera-Ninirola I, et al. Incidencia de cancer en la Region de Murcia. In: Direccion General de Salud Publica, Consejeria de Sanidad de la Region de Murcia, 2007.

16. Breslow NE, Day NE. Statistical methods in cancer research. IARC Workshop 25-27 May 1983. IARC Sci Publ 1987;1-406.

17. Hampel H, Frankel WL, Martin E, et al. Screening for the Lynch syndrome (hereditary nonpolyposis colorectal cancer). N Engl J Med 2005;352:1851-1860.

18. Hampel H, Frankel WL, Martin E, et al. Feasibility of screening for Lynch syndrome among patients with colorectal cancer. J Clin Oncol 2008;26:5783-5788.

19. Boland CR, Shike M. Report from the Jerusalem workshop on Lynch syndrome-hereditary nonpolyposis colorectal cancer. Gastroenterology 2010;138:2197.

20. Chen JM. The 10-Mb paracentric inversion of chromosome arm $2 p$ in activating $\mathrm{MSH} 2$ and causing hereditary nonpolyposis colorectal cancer: re-annotation and mutational mechanisms. Genes Chromosomes Cancer 2008;47:543-545.

21. Guarinos C, Castillejo A, Barbera VM, et al. EPCAM germ line deletions as causes of Lynch syndrome in Spanish patients. J Mol Diagn 2010;12:765-770.

22. Kovacs ME, Papp J, Szentirmay Z, et al. Deletions removing the last exon of TACSTD1 constitute a distinct class of mutations predisposing to Lynch syndrome. Hum Mutat 2009;30:197-203.

23. Ligtenberg MJ, Kuiper RP, Chan TL, et al. Heritable somatic methylation and inactivation of $\mathrm{MSH} 2$ in families with Lynch syndrome due to deletion of the 3' exons of TACSTD1. Nat Genet 2009;41: 112-117.

24. Morak M, Koehler U, Schackert HK, et al. Biallelic MLH1 SNP cDNA expression or constitutional promoter methylation can hide genomic rearrangements causing Lynch syndrome. J Med Genet 2011;48:513-519.

25. Pinheiro M, Pinto C, Peixoto A, et al. A novel exonic rearrangement affecting MLH1 and the contiguous LRRFIP2 is a founder mutation in Portuguese Lynch syndrome families. Genet Med 2011;13:895902.

26. Wagner $\mathrm{A}$, van der $\mathrm{KH}$, Franken $\mathrm{P}$, et al. $\mathrm{A} 10-\mathrm{Mb}$ paracentric inversion of chromosome arm $2 \mathrm{p}$ inactivates $\mathrm{MSH} 2$ and is responsible for hereditary nonpolyposis colorectal cancer in a NorthAmerican kindred. Genes Chromosomes Cancer 2002;35:49-57.

27. Lindor NM, Rabe K, Petersen GM, et al. Lower cancer incidence in Amsterdam-I criteria families without mismatch repair deficiency: familial colorectal cancer type X. JAMA 2005;293:1979-1985.

28. Bonadona V, Bonaiti B, Olschwang S, et al. Cancer risks associated with germline mutations in MLH1, MSH2, and MSH6 genes in Lynch syndrome. JAMA 2011;305:2304-2310. 
29. Carayol J, Khlat M, Maccario J, et al. Hereditary non-polyposis colorectal cancer: current risks of colorectal cancer largely overestimated. J Med Genet 2002;39:335-339.

\section{Appendix. Study Participants: Members of the EPICOLON Consortium (Gastrointestinal Oncology Group of the Spanish Gastroenterological Association)}

Hospital 12 de Octubre, Madrid: Juan Diego Morillas (local coordinator), Raquel Muñoz, Marisa Manzano, Francisco Colina, Jose Díaz, Carolina Ibarrola, Guadalupe López, Alberto Ibáñez; Hospital Clínic, Barcelona: Antoni Castells (local coordinator), Virgínia Piñol, Sergi Castellví-Bel, Francesc Balaguer, Victoria Gonzalo, Teresa Ocaña, María Dolores Giráldez, Maria Pellisé, Anna Serradesanferm, Leticia Moreira, Miriam Cuatrecasas, Josep M. Piqué; Hospital Clínico Universitario, Zaragoza: Ángel Lanas (local coordinator), Javier Alcedo, Javier Ortego; Hospital Cristal-Piñor, Complexo Hospitalario de Ourense: Joaquin Cubiella (local coordinator), $\mathrm{M}^{\text {a }}$ Soledad Díez, Mercedes Salgado, Eloy Sánchez, Mariano Vega; Hospital del Mar, Barcelona: Montserrat Andreu (local coordinator), Anna Abuli, Xavier Bessa, Mar Iglesias, Agustín Seoane, Felipe Bory, Gemma Navarro, Beatriz Bellosillo, Josep $\mathrm{M}^{a}$ Dedeu, Cristina Álvarez, Begoña Gonzalez; Hospital San Eloy, Baracaldo and Hospital Donostia, CIBERehd, University of Country Basque, San Sebastián: Luis Bujanda (local coordinator) Ángel Cosme, Inés Gil, Mikel Larzabal, Carlos Placer, María del Mar Ramírez, Elisabeth Hijona, Jose M. Enríquez-Navascués, Jose L. Elosegui; Hospital General Universitario de Alicante: Artemio Payá (EPICOLON I local coordinator), Rodrigo Jover (EPICOLON II local coordinator), Cristina Alenda, Laura Sempere, Nuria Acame, Estefanía Rojas, Lucía Pérez-Carbonell; Hospital General de Granollers: Joaquim Rigau (local coordinator), Ángel Serrano, Anna Giménez; Hospital General de Vic: Joan Saló (local coordinator), Eduard Batiste-Alentorn, Josefina Autonell, Ramon Barniol; Hospital General Universitario de Guadalajara and Fundación para la Formación e Investigación Sanitarias Murcia: Ana María García (local coordinator), Fernando Carballo, Antonio Bienvenido, Eduardo Sanz, Fernando González, Jaime Sánchez, Akiko Ono; Hospital General Universitario de Valencia: Mercedes Latorre (local coordinator), Enrique Medina, Jaime Cuquerella, Pilar Canelles, Miguel Martorell, José Ángel García, Francisco Quiles, Elisa Orti; CHUVI-Hospital Meixoeiro, Vigo: EPICOLON I: Juan Clofent (local coordinator), Jaime Seoane, Antoni Tardío, Eugenia Sanchez; EPICOLON II: M ${ }^{a}$ Luisa de Castro (local coordinator), Antoni Tardío, Juan Clofent, Vicent Hernández; Hospital Universitari Germans Trias i Pujol, Badalona and Section of Digestive Diseases and Nutrition, University of Illinois at Chicago,
Chicago, IL: Xavier Llor (local coordinator), Rosa M. Xicola, Marta Piñol, Mercè Rosinach, Anna Roca, Elisenda Pons, José M. Hernández, Miquel A. Gassull; Hospital Universitari Mútua de Terrassa: Fernando Fernández-Bañares (local coordinator), Josep M. Viver, Antonio Salas, Jorge Espinós, Montserrat Forné, Maria Esteve; Hospital Universitari Arnau de Vilanova, Lleida: Josep M. Reñé (local coordinator), Carmen Piñol, Juan Buenestado, Joan Viñas; Hospital Universitario de Canarias: Enrique Quintero (local coordinator), David Nicolás, Adolfo Parra, Antonio Martín; Hospital Universitario La Fe, Valencia: Lidia Argüello (local coordinator), Vicente Pons, Virginia Pertejo, Teresa Sala; Hospital Sant Pau, Barcelona: Dolors Gonzalez (local coordinator), Eva Roman, Teresa Ramon, Maria Poca, $\mathrm{M}^{a}$ Mar Concepción, Marta Martin, Lourdes Pétriz; Hospital Xeral Cies, Vigo: Daniel Martinez (local coordinator); Fundacion Publica Galega de Medicina Xenomica (FPGMX), CIBERER, Genomic Medicine GroupUniversity of Santiago de Compostela, Santiago de Compostela, Galicia, Spain: Ángel Carracedo (local coordinator), Clara Ruiz-Ponte, Ceres Fernández-Rozadilla, $\mathrm{M}^{\mathrm{a}}$ Magdalena Castro; Hospital Universitario Central de Asturias: Sabino Riestra (local coordinator), Luis Rodrigo; Hospital de Galdácano, Vizcaya: Javier Fernández (local coordinator), Jose Luis Cabriada; Fundación Hospital de Calahorra (La Rioja) La Rioja: Luis Carreño (local coordinator), Susana Oquiñena, Federico Bolado; Hospital Royo Villanova, Zaragoza: Elena Peña (local coordinator), José Manuel Blas, Gloria Ceña, Juan José Sebastián; Hospital Universitario Reina Sofía, Córdoba: Antonio Naranjo (local coordinator).

Received November 11, 2012. Accepted January 22, 2013.

\section{Reprint requests}

Address requests for reprints to: Rodrigo Jover, MD, Service of Gastroenterology, Hospital General Universitario de Alicante, C/Pintor Baeza 12, 03010 Alicante, Spain. e-mail: jover_rod@gva.es; fax: (34) 965933468.

\section{Acknowledgments}

Writing assistance was provided by SF edits and supported by the Instituto de Salud Carlos III (PI08/0726).

\section{Conflicts of interest}

The authors disclose no conflicts.

\section{Funding}

Supported by grants from Instituto de Salud Carlos III (PI-080726, INT-09/208, and PI11/026030), the Fondo de Investigación Sanitaria/FEDER (PS09/02368, 10/00384, 10/00918, 11/00219, and 11/00681), Fundació Olga Torres (to C.R.-P.) and FP7 CHIBCHA Consortium (S.C.-B. and A. Carracedo), the Ministerio de Economía y Competitividad (SAF2010-19273), and Agència de Gestió d'Ajuts Universitaris i de Recerca (2009 SGR 849). S.C.-B. was supported by a contract from the Fondo de Investigación Sanitaria (CP030070). CIBERER and CIBERehd are funded by the Instituto de Salud Carlos III. 
Supplementary Table 1. Variants of Uncertain Significance (VUS)

\begin{tabular}{|c|c|c|c|c|c|c|c|c|c|}
\hline Patient & Sex & Age $(y)$ & $\mathrm{MSI}$ & $\begin{array}{l}\text { MMR protein } \\
\text { loss }\end{array}$ & Gene & $\begin{array}{c}\text { VUS } \\
\text { (DNA change) }\end{array}$ & Protein change & $\begin{array}{c}\text { Reported insight } \\
\text { (times) }\end{array}$ & $\begin{array}{c}\text { Mainly reported } \\
\text { as }\end{array}$ \\
\hline 1 & Female & 80 & No & MLH1/PMS2 & MLH1 & c. $1013 \mathrm{~A}>\mathrm{G}$ & p.Asn338Ser & 14 & -? (10/14) \\
\hline 2 & Female & 80 & Yes & MLH1/PMS2 & MLH1 & c.1959 G>T & p.Leu653Leu & 20 & $?(11 / 20)$ \\
\hline \multirow[t]{2}{*}{3} & Female & 72 & Yes & MLH1/PMS2 & MLH1 & c.1331 A>G & p.Asn444Ser & Not reported & \\
\hline & & & & & MLH1 & c. $2401 \mathrm{G}>\mathrm{A}$ & p.Ala681Thr & 71 & ?‘ (43/71) \\
\hline 4 & Female & 83 & Yes & MSH2/MSH6 & MSH2 & c. $1021 \mathrm{C}>\mathrm{G}$ & p.Leu341Val & Not reported & \\
\hline 5 & Male & 66 & Yes & MSH2/MSH6 & $\mathrm{MSH} 2$ & c. $366+6 \mathrm{~T}>\mathrm{C}$ & & Not reported & \\
\hline
\end{tabular}

?, variant of uncertain clinical significance; -?, likely not pathogenic. 